\title{
Evaluation of the Function of Ganglion Cells in Myopic Patients Using Pattern Electroretinogram (PERG)
}

\author{
Nasma Nasser*, Yusuf Suleiman, Habib Yousef \\ Ophthalmology Department, Faculty of Medicine, Tishreen University, Lattakia, Syria \\ Email address: \\ Nasmanasser14@gmail.com (N. Nasser),yusuf.suleiman@tishreen.edu.sy (Y. Suleiman), habib.yousef@tishreen.edu.sy (H. Yousef) \\ ${ }^{*}$ Corresponding author
}

\section{To cite this article:}

Nasma Nasser, Yusuf Suleiman, Habib Yousef. Evaluation of the Function of Ganglion Cells in Myopic Patients Using Pattern Electroretinogram (PERG). American Journal of Biomedical and Life Sciences. Vol. 8, No. 6, 2020, pp. 206-211.

doi: $10.11648 /$ j.ajbls.20200806.13

Received: September 30, 2020; Accepted: October 16, 2020; Published: November 23, 2020

\begin{abstract}
Objective: Assessment of the macular ganglion cells function in myopic subjects using pattern electroretinogram (PERG) in comparison with healthy controls to help in diagnosing of retinal diseases, and studying the relationship of the changes seen in PERG with the axial length (AL) of the eye. Methods: The study was cross sectional, included 62 subjects (121 eyes). Three groups were examined: group 1: controls or emmetropics; group 2: mild myopics; group 3: moderate myopics. For all participants, a detailed pathological story was taken, a comprehensive eye examination was performed, AL was measured, and PERG was recorded, then the variables of the recorded waves (peak time and amplitude) were analyzed. Results: A statistically significant decrease in mean amplitudes of (P50, N95) waves was observed in group 2 (36 eyes) and 3 (25 eyes) compared to group 1 (60 eyes) (P-value $<0.01$ ), and a statistically significant elongation of mean N95 peak time was observed in (group 2 and 3) compared to group 1 (P-value $<0.01$ ) without being associated with elongation of mean P50 peak time. By studying the relationship between AL and each of peak time and amplitude of (P50, N95) waves, we have found that when AL increased, amplitudes decreased and peak times prolonged in a statistically significant way (P-value <0.01). Conclusion: The decreased amplitude of P50 wave may indicate a functional impairment of the macula in myopic subjects, and the decreased amplitude of N95 wave may be due to a functional impairment of their ganglion cells. The elongation of P50 peak time with increasing of AL may be due to a delay of the synaptic transmission between photoreceptors, bipolar cells, and ganglion cells.
\end{abstract}

Keywords: Myopia, Pattern Electroretinogram, Axial Length, Macula, Ganglion Cell

\section{Introduction}

Myopia is a worldwide phenomenon, and it is an important social health problem due to its association with an increased risk of vision loss $[1,2]$. The axial elongation of the eye that accompanies myopia results in thinning and decreasing in the density of the retinal pigment epithelium (RPE) in the equatorial region, thinning of choroid and sclera, mostly at the posterior pole [3], and spacing between retinal neurons, including ganglion cells [4]. Also the thickness of ganglion cell- inner plexiform layer (GCIPL) decreased with the increasing of myopic degree and with the increasing of axial length $(\mathrm{AL})$ of the eye $[5,6]$. The previous structural changes are accompanied by functional changes [7]. It's possible to obtain objective information about retinal cells function through visual ectrophysiological tests, including electroretinography (ERG), which are non-invasive tests [8].

The pattern electroretinogram (PERG) helps in understanding the pathophysiology of several eye diseases, and the amplitude of PERG -which represents the ganglion cells function- can detect damage before the conventional field devices, and therefore it is an important predictive factor in patients with high intraocular pressure, it is also a useful tool in the early detection of optic neuropathies such as those occurring in context of ocular Grave's disease (OGD) and the early stages of Alzheimer's disease. It has an important role in determining the pre-proliferative stage in diabetic retinopathy for the possibility of laser treatment, and it helps in explaining of optic nerve diseases if it's compared with the results of pattern reversal visual evoked potentials 


\section{(PRVEP) [9-11].}

Myopia is a risk factor for many retinal diseases that may cause permanent visual damage such as glaucoma and myopic maculopathy, and the current examinations $\{$ (such as fundoscopy and optical coherent tomography (OCT)\} may not be sufficient to differentiate these diseases in their early stages from the normal state of myopia, and because of the importance of early detection of these diseases, we aim through this research to study (PERG) in order to find out its specificity in myopia, by comparing the results with healthy controls, as well as studying the changes that accompany the AL elongation of the eye.

\section{Materials and Methods}

\subsection{Design}

A cross sectional study.

\subsection{Subjects}

Two groups of eye clinic reviewers were examined at Tishreen University Hospital in Lattakia, Syria. From August 2019 till August 2020.

\subsubsection{Ethical Considerations}

An informed consent and ethical committee clearance were taken for this study.

\subsubsection{Inclusion Criteria}

Mypoic subjects with refractions of ( $-0.5 \mathrm{D}$ and less), age group (18-40) years, and emmetropic subjects with refractions between $(-0.25 \mathrm{D}$ and $+0.50 \mathrm{D})$ of the same age group.

\subsubsection{Exclusion Criteria}

Best corrected visual acuity (BCVA) is worth than 20:20, astigmatism $>0.50 \mathrm{D}$, intraocular pressure (IOP) $>21 \mathrm{~mm}$ $\mathrm{Hg}$, blurred media, other ocular diseases such as (uveitis, macular degenerations, etc.), myopic degeneration, previous ophthalmic (surgery, injection or laser treatment), systemic diseases and treatments that may affect electroretinography results, chronic alcoholism, lack of patient cooperation (fatigue and inability to concentrate during the test).

\subsection{Procedures}

Each of (age, social habits, the medical, surgical and pharmacological history) of participants was written in the research questionnaire. All subjects underwent a comprehensive ophthalmic examination (visual acuity, refraction errors, pupil diameter, BCVA, slit-lamp examination, dilated fundoscopy), $\mathrm{AL}$ is measured by Aviso ultrasound A scan (Quantel, Italy) and IOP was measured by Kowa air puff tonometer (KT-800, japan).

PERG was recorded with Retimax (CSO, Italy) in accordance with the International Society for Clinical Electrophysiology of Vision (ISCEV) guidelines [12], general recommendations were given to the participants before the test (sleeping 6-8 hours, avoiding sedatives and hypnotics.). At procedure: The examination method was explained to the participant, the room was quiet, the lighting was dim, and the participant's position was comfortable to avoid muscles spasm and errors. Electrodes were placed (H$\mathrm{K}$ loop electrodes were placed in the fornix of the lower eyelid after topical anesthesia, and referred to ipsilateral outer canthus silver-coated electrodes with a mid-forehead earth electrode), achieving good contact with skin by removing deposits and dead cells using a gentle abrasive tool and a suitable gel between electrode and skin. The interelectrode impedance was checked and maintained below 5 $\mathrm{KOhm}$ in all recordings. The stimulus was black and white cross checkerboard reversing alternately at the rate of $4 \mathrm{rps}$, acquisition time was $250 \mathrm{~ms}$, and the contrast was $99 \%$ "Figure 1". 100 responses were recorded with re-testing twice in the same session.

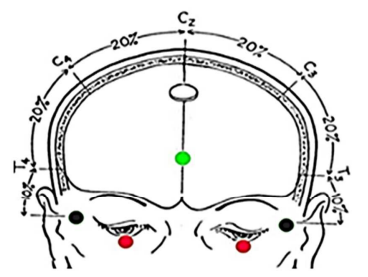

a

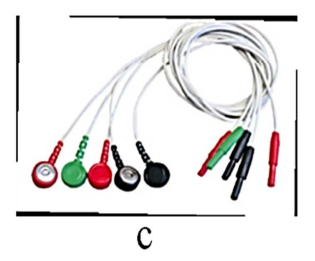

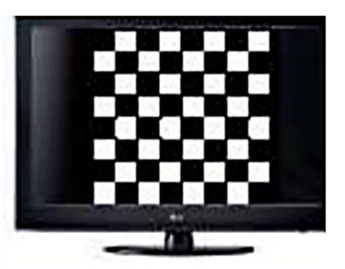

b

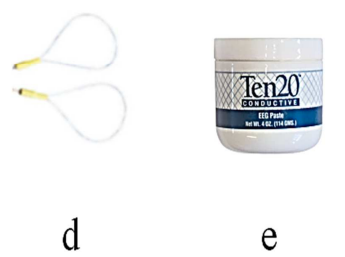

Figure 1. Retimax equipment in the ophthalmological clinic\{(a) the specific electrode contact points for tests; (b) pattern stimulator; (c) silver-coated skin electrodes; (d) H-K loop electrodes; (e) gel; [13].

The test was performed binocularly to facilitate fixation, with undilated pupils, and a corrective glasses suitable for the distance from the screen (1 metre) was used for myopic subjects. Subjects were being instructed to fix their gaze at the sign in center of the checker board, and not to blink, to avoid interference in potentials due to the movement of the eyeball. The pause button was clicked to rest the participant for several seconds to avoid distraction or fatigue, after which the continue button was clicked to complete the recording.

In normal subjects, the PERG wave typically consists of a small primary negative component (may not always appear) with a peak time of about $35 \mathrm{~ms}$ (N35), followed by a much larger positive component (P50) at 45-60 ms, followed by a large negative component (N95) at 90-100 ms [12,13].

The important variables for each wave are recorded: peak time or implicit time which is the time between the start of stimulus and peak response, expressed in milliseconds (ms), and the amplitude which is measured between peaks and troughs. The P50 amplitude is measured from the trough of $\mathrm{N} 35$ to the peak of P50, the N95 amplitude is measured from the peak of P50 to the trough of N95, and so on, often expressed in microvolts $(\mu \mathrm{V})$ [12]. 


\subsection{Statistical Analysis}

Descriptive statistics: the quantitative data (mean value, standard deviation), the qualitative data (frequencies, percentile). We use One Way Anova test and Chi-Square test as inferential statistics. Pearson Correlation was used to study the correlation between the variables. A P-value less than 0.05 was considered statistically significant. IBM SPSS statistics version 20 was used for data analysis.

\section{Results}

The study included 62 participants (121 eyes). Myopics were 32 subjects (61 eyes), they were divided into two groups \{mild myopics (36 eyes) and moderate myopics ( 25 eyes) $\}$. Emmetropics or controls were 30 subjects (60 eyes). The median age of the participants was $26.03 \pm 3.8$ years.
Three groups were formed according to their spherical equivalent (SE) values:

Group 1 (control group): between -0.25 and $+0.50 \mathrm{D}$

Group 2 (mild myopia): between -0.50 and $-2.99 \mathrm{D}$

Group 3 (moderate myopia): between -3.00 and $-5.99 \mathrm{D}$

The (gender, age, SE and $\mathrm{AL}$ ) of the three groups are presented in Table 1. There was no statistical differences in gender and age between the three groups, while there was a statistically significant difference between the groups in mean $\mathrm{AL}$ values which were found longer in myopics.

The results have shown a statistically significant differences in mean N95 peak times between the three groups, but there was no statistical significant differences in mean P50 peak times (Table 1).

There was a statistically significant differences in mean (P50 and N95) amplitudes between the three groups (Table 1; Figure 2).

Table 1. The means obtained for different parameters among the three groups.

\begin{tabular}{llll}
\hline \multirow{2}{*}{ Study subjects } & Group 1 (60 eyes) & Myopics (61 eyes) & Group 3 (25 eyes) \\
\cline { 3 - 4 } & & Group 2 (36 eyes) & $9 / 5$ \\
\hline Gender $(\mathrm{M} / \mathrm{F})$ & $8 / 22$ & $11 / 10$ & $25 \pm 3.4$ \\
Age (year) & $26.4 \pm 2.8$ & $24.8 \pm 4.3$ & $-3.4 \pm 0.4$ \\
Spherical equivalent $(\mathrm{D})$ & $0.1 \pm 0.2$ & $-1.4 \pm 0.6$ & $25.1 \pm 0.8$ \\
Axial length $(\mathrm{mm})$ & $23.3 \pm 0.9$ & $24.1 \pm 0.6$ & $50.4 \pm 1.6$ \\
P50 peak time $(\mathrm{ms})$ & $49.8 \pm 1.6$ & $50.2 \pm 1.4$ & $99.8 \pm 8.3$ \\
N95 peak time $(\mathrm{ms})$ & $95.5 \pm 5.8$ & $99.04 \pm 7.8$ & $4.6 \pm 1.1$ \\
P50 amplitude $(\mu \mathrm{V})$ & $6.1 \pm 1.3$ & $5.2 \pm 1.2$ & 0.0001 \\
N95 amplitude $(\mu \mathrm{V})$ & $9.4 \pm 1.1$ & $7.3 \pm 1.2$ & 0.0 \\
\hline
\end{tabular}

D: Diopter, mm: millimeter, ms: millisecond, $\mu \mathrm{V}$ : microvolt.

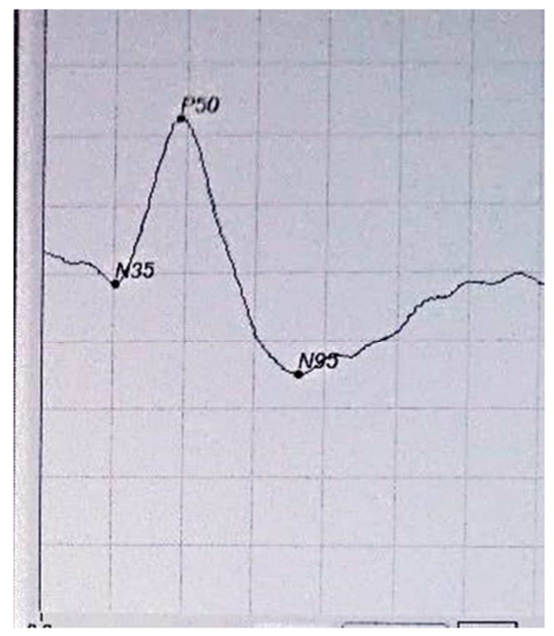

a

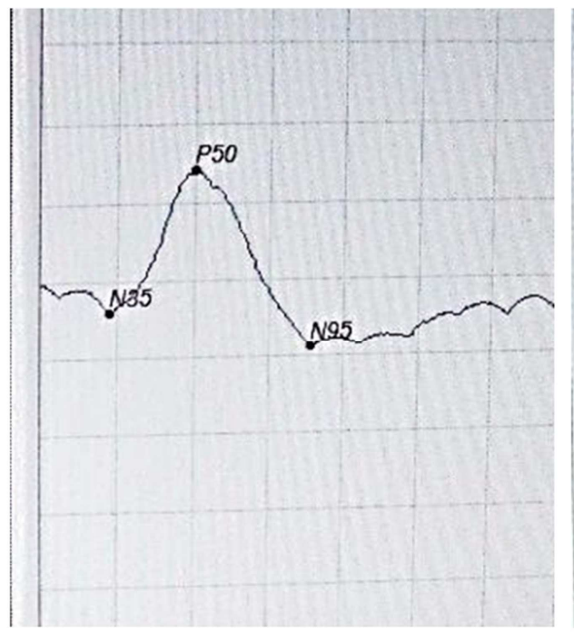

b

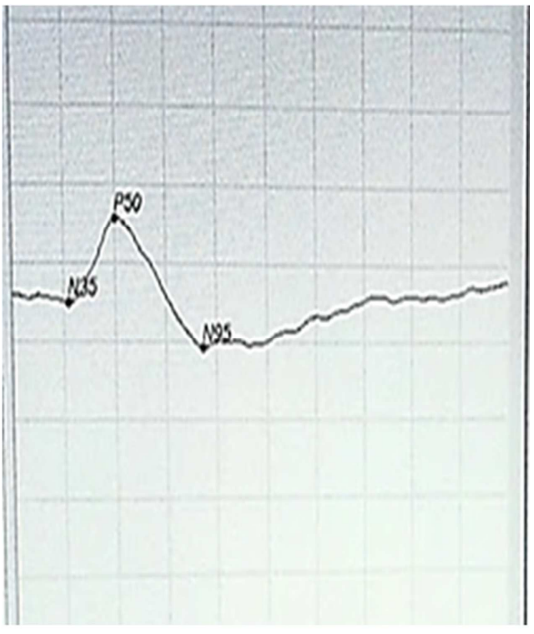

C

Figure 2. Samples of PERG waves: \{(a) controls, (b) mild myopia, (c) moderate myopia\}.

By studying the relationship between AL and each of (P50, N95) peak times and amplitudes, it was found that when AL increased, peak times prolonged and amplitudes decreased in a statistically significant way (Table 2). There was no statistically significant correlation between SE and P50 peak time, but it was a statistically significant negative correlation between SE and N95 peak time. When P50 and N95 amplitudes were correlated to SE, it was found that when SE decreased, the amplitudes decreased in statistically significant way (Table 3).

Table 2. Correlation of PERG parameters with AL.

\begin{tabular}{lll}
\hline PERG parameters & r & P-value \\
\hline P50 peak time $(\mathrm{ms})$ & 0.46 & 0.001 \\
N95 peak time $(\mathrm{ms})$ & 0.31 & 0.002 \\
P50 amplitude $(\mu \mathrm{V})$ & -0.39 & 0.001 \\
N95 amplitude $(\mu \mathrm{V})$ & -0.51 & 0.0001 \\
\hline
\end{tabular}

ms: millisecond, $\mu \mathrm{V}$ : microvolt. 
Table 3. Correlation of PERG parameters with SE.

\begin{tabular}{lll}
\hline PERG parameters & r & P-value \\
\hline P50 peak time $(\mathrm{ms})$ & -0.21 & 0.05 \\
N95 peak time $(\mathrm{ms})$ & -0.29 & 0.01 \\
P50 amplitude $(\mu \mathrm{V})$ & 0.41 & 0.0001 \\
N95 amplitude $(\mu \mathrm{V})$ & 0.63 & 0.0001 \\
\hline
\end{tabular}

ms: millisecond, $\mu \mathrm{V}$ : microvolt.

\section{Discussion}

PERG is a non-invasive electrophysiological ophthalmic test that provides a quantitative measurement of central retinal function, and measures objectively the functional impairment and recovery. Since it is a localized response from the area covered by a checkerboard image, it can be used as an indicator of the dysfunction within the macula region, noting that black and white exchange is the most important induction of the contrast response for ganglion cells $[12,14]$.

Our study agreed with the study of Ayse Oner et al [15], where 4 groups were formed according to the refraction values of 160 eyes of 80 myopic and control subjects as follows: Group 1 (controls): between 0.00 and $-0.75 \mathrm{D}$; Group 2: -1.00 and -3.00 ; Group 3: -3.25 and $-6.00 \mathrm{D}$; Group 4: -6.25 and -10.00 D. There was no statistically significant differences between groups in age and sex, but there was a statistically significant positive correlation between myopia and AL. Also, it's found a statistically significant prolongation in mean N95 peak time in subjects whom SE values were less than - 3.00 D, but no statistically significant difference was found in mean P50 peak time. The amplitudes of P50 and N95 were statistically significant decreasing with the decreasing in values of SE and with the increasing in AL. However it didn't evaluate the correlation between P50 and N95 peak times and both of SE and AL.

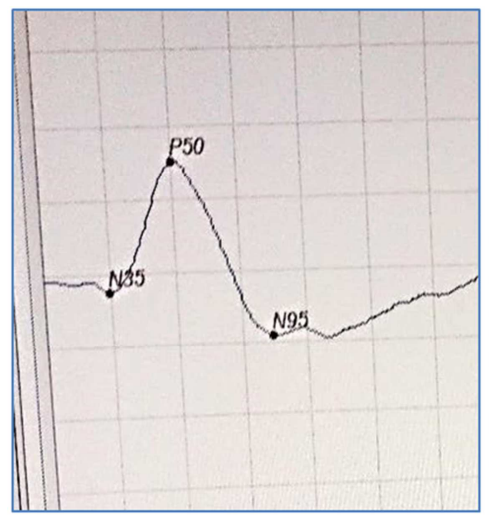

Figure 3. Shows the wide shape of $N 95$.

Prolongation of peak time in myopics compared to controls may indicate differences in the kinetics of synaptic transport from photoreceptors to the On and Off pathways of bipolar cells and ganglion cells [19]

As for Amplitude (which is the maximum electrical response evoked by light and generated by retinal cells), low P50 amplitude in myopic eyes compared to
In another study by L K Sumitra et al [16], where total of 64 subjects were included in the study of 18-30 years age group of both sexes. The subjects were divided into 4 groups as the same way as Ayse Oner study did, it's shown that the mean (P50 and N95) amplitudes were less in myopic groups than in control group, also the amplitudes decreased with increase in refractive error and axial length $(p<0.001)$, it has found a statistically significant prolongation of mean N95 peak time in myopics, the previous are consistent with our study, but L K Sumitra differed from our study when it's found a statistically significant prolongation of mean P50 peak time in myopic groups compared to control group, this could be due to the difference of mean SE value in mild and moderate myopic groups which was $-1.4 \pm 0.6$ and $-3.4 \pm 0.4$ $\mathrm{D}$ respectively in our study while in $\mathrm{L} \mathrm{K}$ Sumitra study it was $-2.12 \pm 0.52$ and $-4.7 \pm 0.74 \mathrm{D}$ respectively, in addition to the presence of the severe myopic group in the comparison between groups in L K Sumitra study while it didn't present in our study.

Our study also agreed with that of Rudy Hidajat et al [17], which included Thirty-nine normal volunteers with AL ranged between 21.8 and $25.7 \mathrm{~mm}$, where it's found a significant negative correlation between $\mathrm{AL}$ and P50 amplitude.

Several hypotheses have been proposed regarding to PERG findings in our study, the spacing occurred between retinal neurons including ganglion cells, and the thining of ganglion cell-inner plexiform layer (GCIPL) due to an increase in (AL or myopic refraction) contribute to the decrease in ERG potentials in myopic eyes [5-7].

Regarding N95 peak time, although we've found a statistically significant prolongation in myopic groups comparing to controls, it is likely that it has no clinical value, because it's not easy to measure N95 peak time accurately due to its wide shape [18] "Figure 3".

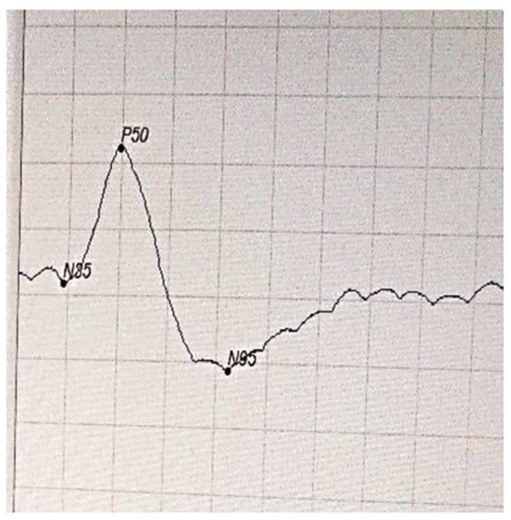

emmetropics could be due to a decrease in the density of the photoreceptors, changes in the morphology of their external part, and the disturbance in the function of these receptors in the macula [20]. N95 amplitude represents a direct measure of the ganglion cells function and its decrease in our study may indicate a lack of its function in the myopia groups [21]. 
The dopaminergic system may contributes in explanation of the altered responses in myopia, dopamine molecules have receptors within the retina, especially the ON pathway for photoreceptors, bipolar cells and ganglion cells. Dopamine contributes in reducing ocular elongation and myopia development $[22,23]$, it also participates in retinal cell survival and in reorganizing the properties of the receptive fields associated with changes in retinal illumination, and modifies the kinetic properties of the ganglion cell response to contrast. A deficiency in dopamine levels- as occurs in Parkinson's patients- leads to a significant decrease in (P50 and N95) amplitudes with a delayed in P50 peak time [24, 25].

\section{Conclusion}

The mean values of (P50, N95) amplitudes decreased in myopic groups compared to emmetropic group, and the decrease increased as SE value decreased. As AL increased, amplitudes decreased and peak times prolonged for each P50 and N95 waves. These changes in PERG indicate a disturbance in the function of the macula and the ganglion cells in myopics compared to emmetropics, despite the presence of a normal eye fundus.

PERG is recommended for myopics when degrees stabilize, in order to have a reference database to return to in case of any central retinal lesion with a normal fundus is suspected.

\section{Conflicts of Interest}

All the authors do not have any possible conflicts of interest.

\section{References}

[1] Hopf, S; Pfeiffer N." Epidemiology of myopia. " Ophthalmologe, 2017; 114/1: 20-23.

[2] Chu, H; Cheng, C. Optimal social security tax with myopic agents. Economic Modelling, Vol 80, 2019, 320-327.

[3] Jonas, J. B; Jonas, S. P. "Epidemiology and anatomy of myopia."Ophthalmologe, 2019; 116/6: 499-508.

[4] Coletta, N. G; Watson, T. "Effect of myopia on visual acuity measured with laser interference fringe." Vision Research, 2006; 46/5: 636-51.

[5] Shpak, A. A; Korobkova, M. V. "Causes of ganglion cellinner plexiform layer thinning in myopic eyes." Graefes Arch Clin Exp Ophthalmol, 2020; 258/1: 3-7.

[6] Takeyama, A; Kita, Y; Kita, R; Tomita, G. "Influence of axial length on ganglion cell complex (GCC) thickness and on GCC thickness to retinal thickness ratios in young adults."Japanese Journal of Ophthalmology, 2014; 58/1: 86-93.

[7] Ismael, Z. F; El-Shazly, A. A; Farweez, Y. A; Osman, M. M."Relationship between functional and structural retinal changes in myopic eyes."Clinical and Experimental Optometry, 2017; 100/6: 695-703.
[8] Robson, A. G; Nilsson, J; Li, S; Jalali, S; Fulton, A. B; Tormene, A. P; Holder, G. E; Brodie, S. E. " ISCEV guide to visual electrodiagnostic procedures." Doc Ophthalmol, 2018; 136/1: 1-26.

[9] Bach, M; Sulimma, F; Gerling, J." Little correlation of the pattern electroretinogram (PERG) and visual field measures in early glaucoma." Doc Ophthalmol, 1998; 94/3: 253-63.

[10] Krasodomska, K; Lubiński, W; Potemkowski, A; Honczarenko, K. "Pattern electroretinogram (PERG) and pattern visual evoked potential (PVEP) in the early stages of Alzheimer's disease." Doc Ophthalmol, 2010; 121/2: 111-121.

[11] Benedek, K; Pálffy, A; Bencsik, K; Fejes, I; Rajda, C; Tuboly, G; Liszli, P. "[Combined use of pattern electroretinography and pattern visual evoked potentials in neuroophthalmological practice]." Ideggyogy Sz, 2008; 61/(1-2): 33-41.

[12] Bach, M; Brigell, M. G; Hawlina, M; Holder, G. E; Johnson, M. A; McCulloch, D. L; Meigen, T; Viswanathan, S. "ISCEV standard for clinical pattern electroretinography (PERG): 2012 update." Doc Ophthalmol, 2013; 126: 1-7.

[13] Instructions for Use and Maintenance - SISTEMA RETIMAX. retimographOPHT- 06-User Manual \& Service Maual.

[14] Asanad, S; Karanjia, R." Pattern Electroretinogram (PERG)." StatPearls Publishing US, Jan 2020.

[15] Oner, A; Gumus, K; Arda, H; Karakucuk, S; Mirza, E. Pattern electroretinographic recordings in eyes with myopia. Erciyes Medical Journal, Vol. 33 No. 1 pp, 2011, 17-22.

[16] Sumitra, L. K; Kumari, A. S; Rajkumari, C. N. Electroretinogram Changes in Adult Individuals with Myopia. International Journal of Physiology India, Vol. 4, Issue: 1, 2016, 32-38.

[17] Hidajat, R; Mclay, J; Burley, C; Elder, M; Morton, J; Goode, D. Influence of axial length of normal eyes on PERG. Documenta Ophthalmologica, Vol. 107, 2003, 195-200.

[18] Holder, G. E. "Pattern electroretinography (PERG) and an integrated approach to visual pathway diagnosis. " Progress in Retinal and Eye Research, 2001; 20/4: 531-61.

[19] DWIGHT, B. A. Contrast processing by ON and OFF bipolar cells. Visual Neuroscience Cambridge, Vol. 28, Iss. 1, 2011, 69-75.

[20] Hood, D. C. Assessing retinal function with the multifocal technique. Progress in Retinal and Eye Research, Vol. 19, Issue 5, 2000, 607-646.

[21] Brar, V. S; Law, S. K; Lindsey, J. L; Mackey, D. A; Schultze, L. R; Singh, R. S; Silverstein, E. American Academy of Ophthalmology, Basic and Clinical Science Course, retina and vitreous. $2^{\text {nd }}$ ed, European Board of Ophthalmology, Netherlands, 2019-2020, 569.

[22] Landis, E. G; Chrenek, M. A; Chakraborty, R; Strickland, R; Bergen, M; Yang, V; Iuvone, P. M; Pardue, M. T. Increased endogenous dopamine prevents myopia in mice. Experimental Eye Research, Vol. 193, 2020, 107956.

[23] Chen, S; Zhi, Z; Ruan, Q; Liu, Q; Li, F; Wan, F; Reinach, P. S; Chen, J; Qu, J; Zhou, X. Bright Light Suppresses FormDeprivation Myopia Development With Activation of Dopamine D1 Receptor Signaling in the ON Pathway in Retina. Investigative Ophthalmology \& Visual Science, Vol. 58, 2017, 2306-2316. 
[24] Witkovsky, P. Dopamine and retinal function. Documenta Ophthalmologica, Vol. 108, 2004, 17-39.

[25] Martin, E. G; Mena, D. R; Satue, M; Almarcegui, C; Dolz, I;
Alarcia, R; Seral, M; Polo, V; Larrosa, J. M; Pablo, L. E. Electrophysiology and Optical Coherence Tomography to Evaluate Parkinson Disease Severity. Investigative Ophthalmology \& Visual Science, Vol. 55, 2014, 696-705. 